\title{
Cerebral Vasospasm Manifesting as a Transient Lesion of the Splenium of the Corpus Callosum Secondary to Pituitary Apoplexy
}

Jocelyn Y. Cheng ${ }^{1 *}$ and Tooba Fayyaz ${ }^{2}$

${ }^{1}$ NYU Comprehensive Epilepsy Center, NYU Department of Neurology, NYU School of Medicine, New York, NY 10016, USA

${ }^{2}$ Cape Regional Physicians Associates, Cape May Court House, NJ 08210, USA

Correspondence to:

Jocelyn Y. Cheng

NYU Comprehensive Epilepsy Center

NYU Department of Neurology

NYU School of Medicine

New York, NY 10016, USA

Tel: 646-558-0797

E-mail: jocelyn.cheng@nyumc.org

Received: December 13, 2017

Accepted: January 16, 2018

Published: January 17, 2018

Citation: Cheng JY, Fayyaz T. 2018. Cerebral Vasospasm Manifesting as a Transient Lesion of the Splenium of the Corpus Callosum Secondary to Pituitary Apoplexy. J Neuroimaging Psychiatry Neurol 2(2): 43-47.

Copyright: (C) 2018 Cheng and Fayyaz. This is an Open Access article distributed under the terms of the Creative Commons Attribution 4.0 International License (CC-BY) (http:// creativecommons.org/licenses/by/4.0/) which permits commercial use, including reproduction, adaptation, and distribution of the article provided the original author and source are credited.

Published by United Scientific Group

\begin{abstract}
Background: Magnetic resonance imaging (MRI) abnormalities isolated to the splenium of the corpus callosum represent a unique radiologic phenomenon. The differential diagnosis includes toxic/metabolic dysfunction, demyelinating disease, seizures, trauma, tumor, infection and vasospasm. Though vasospasm may occur in multiple settings, splenial lesions associated with vasospasm secondary to hemorrhagic pituitary apoplexy have not been well described.
\end{abstract}

Methods: Case report, with review of clinical and radiologic data.

Results: A 50-year-old-right-handed-man presented with decreased visual acuity, right retro-orbital headache and right-sided ptosis over two weeks, followed by complete vision loss in the right eye two days prior to admission. Initial MRI brain revealed findings consistent with hemorrhagic pituitary apoplexy, as well as a non-enhancing lesion of the splenium, which demonstrated diffusion restriction on diffusion weighted imaging with apparent diffusion coefficient correlation, and which was hypointense on T1 and hyperintense on T2. MR spectroscopy excluded neoplasm and demyelinating disease. CTA of the head and neck followed by confirmatory digital subtraction angiography demonstrated narrowing of the parasellar arteries, consistent with vasospasm. The patient was treated with nimodopine and dexamethasone, and post-operative MRI after transnasal transphenoidal resection showed resolution of the splenial lesion.

Conclusion: Lesions isolated to the splenium may represent cerebral vasospasm. Early recognition is essential and should trigger further investigation, as cerebral vasospasm and pituitary apoplexy represent neurological emergencies in which prognosis can be favorable with early and appropriate management.

\section{Keywords}

Cerebral vasospasm, Pituitary apoplexy, Splenium, MRI, Diffusion weighted imaging, Corpus callosum

\section{Abbreviations}

MRI: Magnetic Resonance Imaging; MRS: Magnetic Resonance Spectroscopy

\section{Introduction}

Magnetic resonance imaging (MRI) abnormalities isolated to the splenium of the corpus callosum represent a unique radiologic phenomenon. Posited etiologies include toxic/metabolic dysfunction, seizures, trauma, infection and vasospasm [1]. While vasospasm has been described in multiple settings, 
including pituitary apoplexy, the manifestation of vasospasm as an isolated splenium lesion due to pituitary apoplexy has not been previously described. Herein, we report the case of a patient with a transient lesion of the splenium of the corpus callosum secondary to vasospasm from pituitary adenoma hemorrhage, prior to surgical intervention.

\section{Materials and Methods}

Case report, with review of clinical and radiologic data. The institution waived consent secondary to the retrospective nature of the study.

\section{Results}

A 50-year-old-right-handed-man with no significant past medical history presented with decreased visual acuity, right retro-orbital headache and right-sided ptosis over two weeks, followed by complete vision loss in the right eye two days prior to admission. There was no history of altered consciousness, seizures, preceding illness, alcohol and substance abuse, hypertension, or head trauma. He did not take any medications or over the counter drugs.

On examination, the right eye demonstrated proptosis, with an afferent pupillary defect, palsies of cranial nerves II and III, and absent light perception. Visual acuity in the left eye was $20 / 70$, with a temporal hemianopia. There were no signs or symptoms of a disconnection syndrome, and reading and writing were intact. Examination of sensation, motor function and coordination was normal. Laboratory evaluation was unremarkable, with normal electrolytes levels, including sodium and glucose, normal ESR and CRP, and negative ANA panel and drug and alcohol testing. Blood pressure at presentation was $135 / 71$, and he remained normotensive for the duration of his hospital course.

Initial MRI brain revealed a $2.2 \times 1.7 \times 1.5 \mathrm{~cm}$ sellar mass which was hyperintense on $\mathrm{T} 1$, demonstrated signal loss on gradient echo, and showed mild contrast enhancement, consistent with hemorrhagic pituitary apoplexy (Figure 1). Minimal subarachnoid hemorrhage was present. The optic chiasm was mildly elevated. A non-enhancing lesion of the splenium was also noted, which demonstrated diffusion restriction on diffusion weighted imaging (Figure 2) with apparent diffusion coefficient correlation, and which was hypointense on $\mathrm{T} 1$ and hyperintense on $\mathrm{T} 2$.

Further investigation included magnetic resonance spectroscopy (MRS), which excluded neoplasm and demyelinating disease, as there was no significant abnormality in the choline or $\mathrm{N}$-acetylaspartate peaks. Magnetic resonance angiography and computed tomography angiography of the head and neck revealed diffuse parasellar internal carotid narrowing suggestive of vasospasm. Subsequent digital subtraction angiography demonstrated severe narrowing of the right parasellar internal carotid artery (Figure 3) and moderate narrowing of the left parasellar artery, confirming the presence of vasospasm.

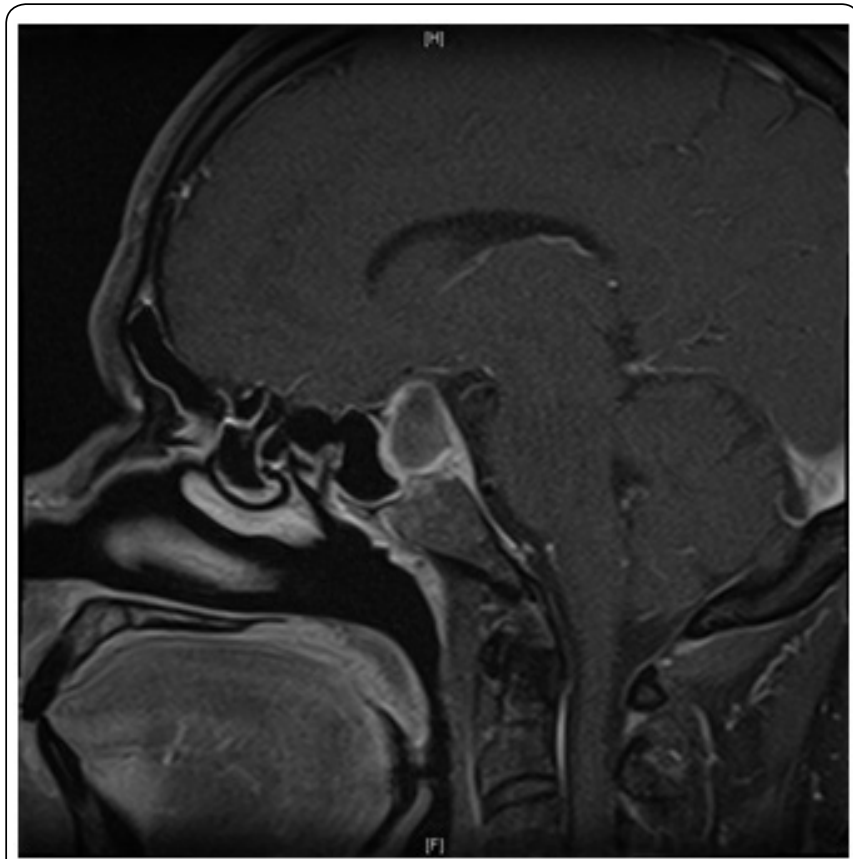

Figure 1: Sagittal T1 MRI brain with contrast.

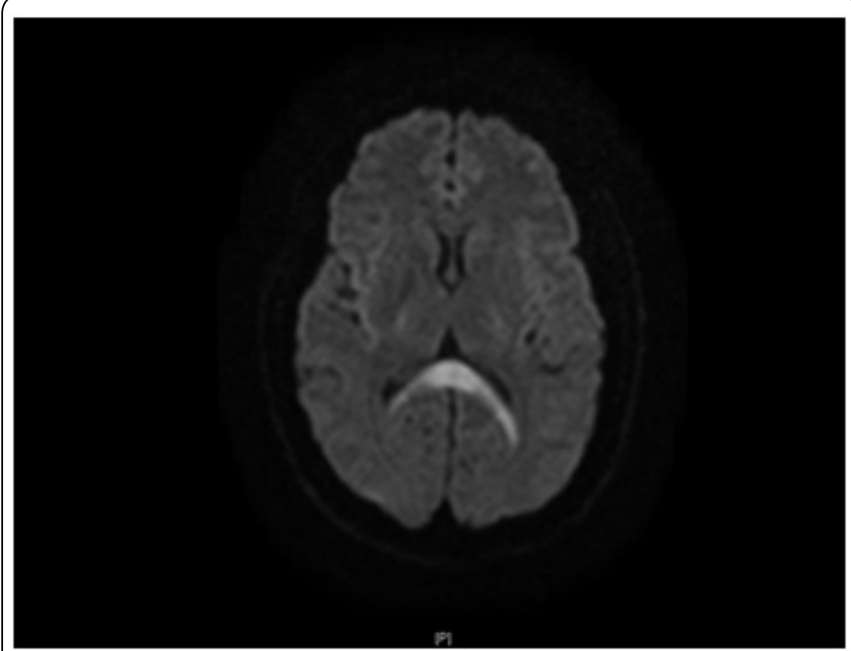

Figure 2: MRI brain, axial diffusion weighted imaging.

The patient was started on nimodipine for vasospasm, and high dose dexamethasone for edema related to the pituitary mass. Visual acuity improved to $20 / 800$ in the right eye and $20 / 25$ in the left eye, with resolution of the temporal hemianopia.

Four days after admission, repeat brain MRI demonstrated improvement of restricted diffusion in the splenium of the corpus callosum. On hospital day twelve, the patient underwent transnasal transsphenoidal resection for tumor evacuation (Figure 4). Pathology revealed fragments of necrotic tissue with acute inflammation and hemorrhage consistent with a pituitary tumor. Post-operative MRI showed resolution of the splenium diffusion abnormality (Figure 5).

\section{Discussion}

This case highlights the importance of recognizing that an 
isolated MRI lesion of the splenium may represent vasospasm in the setting of pituitary apoplexy.

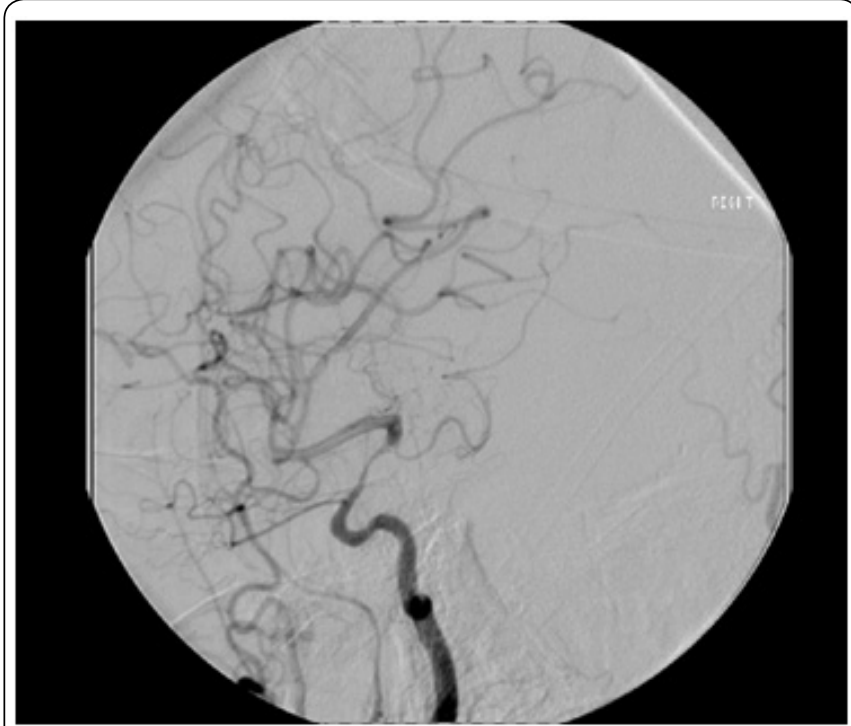

Figure 3: Digital subtraction angiography demonstrating vasospasm of the right parasellar internal carotid artery.

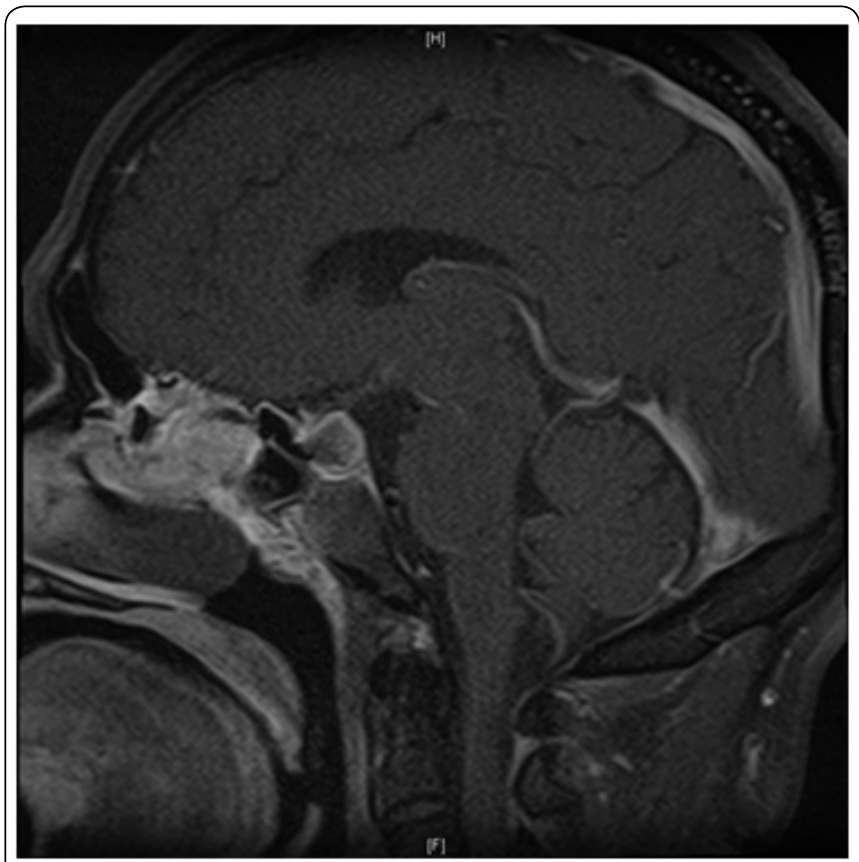

Figure 4: Post-operative sagittal T1 MRI brain with contrast.

Splenial lesions have been described in many different clinical scenarios. Etiologies include vascular, demyelinating, inflammatory, traumatic, neoplastic, infectious, and toxic/ metabolic processes. Vascular lesions may be due to infarction, and are often lateralized to one side due to the bilateral arterial supply of the corpus callosum, but rare midline lesions have been described due to watershed involvement between both anterior or both posterior cerebral arteries, or from occlusion of a third small artery originating from the anterior communicating artery, which may only be detected postmortem. Small vessel disease, such as cerebral autosomal dominant arteriopathy with subcortical infarcts

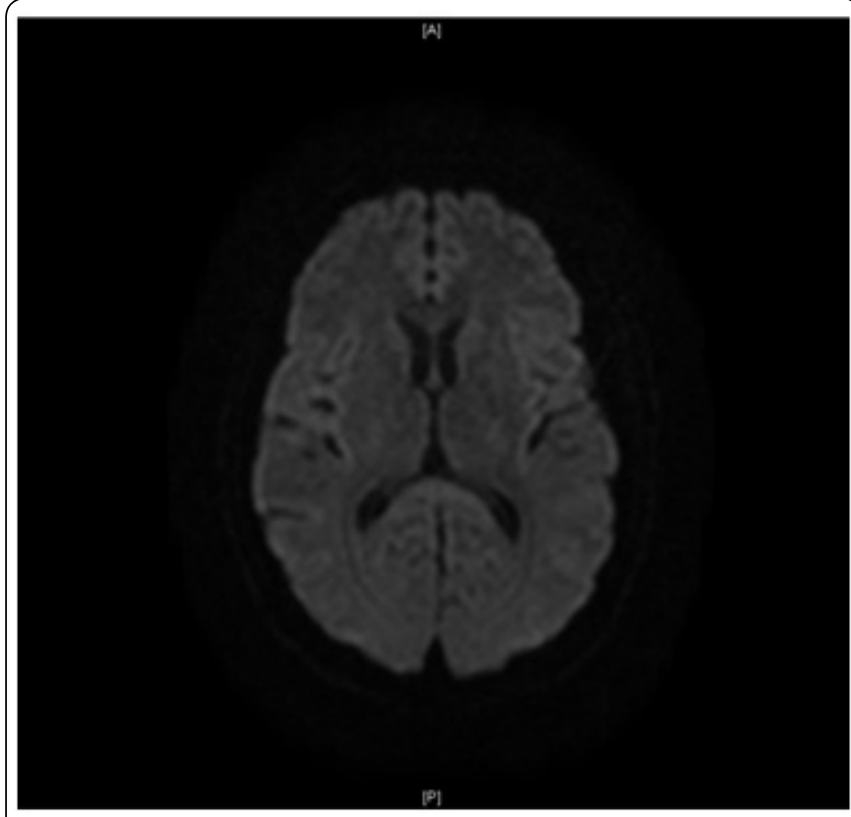

Figure 5: Post-operative MRI brain, axial diffusion weighted imaging.

and leukoencephalopathy (CADASIL) and Susac's syndrome, may also be a cause, although multifocal lesions are typically present. Demyelinating diseases include multiple sclerosis (MS), acute disseminated encephalomyelitis (ADEM), and less frequently, neuromyelitis optica (NMO), although $\mathrm{NMO}$ is more likely to involve the splenium compared to MS and ADEM. Non-demyelinating inflammatory diseases (i.e. Sjögren syndrome, lupus erythematosis, sarcoidosis) can present with splenial lesions in conjunction with more commonly found periventricular white matter lesions. In the setting of trauma, injury involves regions of the brain where differing densities move relative to one other due to changes in rotation and velocity, resulting in the deformation and tearing of axons, aka diffuse axonal injury. Typically affected regions include the posterior more than anterior corpus callosum, midbrain and lobar white matter. Of the neoplastic diseases affecting the central nervous system, glioblastoma multiforme, gliomatosis cerebri and lymphoma more commonly involve the splenium. Various viral encephalitidies have been reported with corpus callosal lesions, including influenza, EBV, HSV-6, and JCV, the latter of which may cause progressive multifocal leukoencephalopathy. Abscesses may also present similarly. In terms of metabolic disease, Marchiafava-Bignami (associated with chronic alcoholism and vitamin deficiency), Wernicke's encephalopathy (as from thiamine deficiency), and osmotic demyelination (as from rapid correction of hyponatremia) may result in splenial lesions. Toxicity from immunosuppressive agents, antimicrobials, illicit drugs, antiepileptic drug withdrawal and environmental toxins are alternative metabolic causes. Transient splenial lesions may also be a consequence of posterior reversible encephalopathy syndrome, the etiology of which includes hypertension and toxic/metabolic disturbance, radiation induced leukoencephalopathy, and recent seizures. In-born errors of metabolism and hereditary leukodystrophies may also involve the splenium, but diffuse involvement of other regions is present as well [1]. 
Although the most common presentation of splenial lesions is altered mental status, other symptoms include confusion, ataxia, seizure, hemispheric disconnection findings, and dysarthria [2]. The absence of neurological symptoms has also been described, despite persistence of the focal lesion [3].

Splenial lesions may persist for a variable amount of time, with most resolving in a few months. Brain MRI typically shows non-enhancing lesions with restricted diffusion and hyperintensity on $\mathrm{T} 2$ images.

In our patient, the most likely etiology for the splenial lesion was vasospasm secondary to extravasation of blood products into the subarachnoid space from hemorrhagic pituitary apoplexy. Alternative causes were excluded by history, in which there were no clinical features suggestive of pre-existing leukodystrophy or errors of in-born metabolism, preceding infection, seizure, hypertension, and trauma, including surgical manipulation; laboratory studies, which did not demonstrate electrolyte imbalance, substance abuse, or evidence for inflammation or infection; and further radiological evaluation, as MRS did not show evidence for neoplasm or demyelination, and angiography was not consistent with arteriopathy or vascular occlusion suggestive of ischemic stroke. The results of this diagnostic evaluation, in conjunction with angiography consistent with vasospasm, suggests that vasospasm due to subarachnoid blood from hemorrhagic pituitary apoplexy was the most likely culprit for our patient's splenial lesion.

While pituitary apoplexy has been associated with vasospasm in the literature, particularly in the post-operative setting, the exact mechanism remains unclear. Hypothalamic damage is often observed with pituitary apoplexy and has been reported to cause vasospasm. There is evidence that the amount of subarachnoid extravasation of blood from the pituitary hemorrhage is related to the degree of vasospasm. Supporting this, cases of vasospasm after pituitary manipulation in the operating room have often been reported, commonly in association with the presence of blood products, with subsequent ischemic changes. Three cases of delayed cerebral vasospasm after transsphenoidal resection of pituitary adenomas complicated by hemorrhage were reported, with MRI confirmed acute ischemic changes in the bilateral anterior cerebral artery territories, and in one case, also involving the left posterior cerebral artery [4]. In a different series, transsphenoidal pituitary surgery complicated by subarachnoid hemorrhage resulted in vasospasm. MRI evidence of acute ischemia was associated with some, but not all, of these cases, with the one reported example of diffusion restriction occurring in the territory of the right middle cerebral artery [5]. Another case of pituitary apoplexy resulted in bilateral carotid vasospasm and right parietal infarct, in the absence of surgical manipulation [6]. Cerebral vasospasm has also been reported as a consequence of meningitis after transsphenoidal pituitary surgery, although in this series, the presence of subsequent ischemic changes on neuroimaging was not assessed [7].

Overall, cerebral vasospasm after pituitary apoplexy or surgery is not an uncommon phenomenon. However, unique to the present case is the finding of transient ischemic changes isolated to the splenium of the corpus callosum.

In our patient, angiography demonstrated signs of vasospasm in the parasellar arteries (i.e. anterior circulation) rather than the vertebrobasilar system, which supplies the splenium. However, circulation of hemorrhagic products likely precipitated vasospasm. Supporting this hypothesis, subsequent brain MRIs demonstrated resolving diffusion restriction of the splenium after nimodipine was started but prior to surgical intervention, and during which time circulating hemorrhagic products were resorbed.

The splenium has a unique blood supply in that it is supplied by the vertebrobasilar system rather than the internal carotid arteries, which supply the rest of the corpus callosum. The vertebrobasilar system has been shown to be more vulnerable to changes in perfusion pressure than the anterior circulation in many clinical conditions such as reversible posterior leukoencephalopathy syndrome and eclampsia [8]. The most commonly accepted theory concerns the difference in autoregulation in the posterior circulation as compared to the anterior circulation. Breakdown in cerebral autoregulation is more commonly seen in the posterior system, as there is a relative lack of sympathetic innervation compared to the anterior system [9]. An alternate hypothesis that has been proposed for the differences in the autoregulatory mechanisms of the anterior and posterior system is that they operate under different metabolic states [8].

The initial work up of a patient presenting with a transient lesion isolated to the splenium should include a full history and physical, and laboratory evaluation to rule out toxic/metabolic derangements, inflammatory disease, and infections, and an MRI evaluation with and without contrast to help distinguish between different causes of splenial lesions, including but not limited to demyelinating disease, tumors, MarchiafavaBignami disease, posterior reversible encephalopathy syndrome, leuokodystrophy, and strokes. Angiography should also be performed to assess for, and appropriately manage, potential vasospasm, which may result in cerebral ischemia and infarction.

The prognosis of transient splenial lesions is favorable unless the underlying etiology is severe. Recognition and treatment of the underlying cause is essential. In our patient, the transient splenial lesion was secondary to vasospasm from hemorrhagic pituitary apoplexy. In the case of pituitary apoplexy, immediate treatment with corticosteroids is mandated, and unless there is a contraindication, surgical resection is indicated. Prognosis is often favorable. Postoperatively, many patients will continue to have endocrine abnormalities, especially in the case of residual tumor, and close follow up is necessary [5].

\section{Conclusion}

We present a novel case of vasospasm presenting as a transient lesion of the splenium of the corpus callosum due to hemorrhagic pituitary apoplexy. Recognition that an 
isolated splenial lesion may represent vasospasm is essential, and should trigger further investigation, as cerebral vasospasm and pituitary apoplexy represent neurological emergencies in which prognosis can be favorable with early and appropriate management.

\section{Conflict of Interest}

The authors declare no conflict of interest.

\section{Acknowledgements}

Acquisition, analysis of data, and drafting and approval of the manuscript and figures were performed by Jocelyn Y. Cheng, MD and Tooba Fayyaz, DO.

\section{References}

1. Renard D, Castelnovo G, Campello C, Bouly S, Le Floch A, et al. 2014. An MRI review of acquired corpus callosum lesions. J Neurol Neurosurg Psychiatry 85(9): 1041-1048. https://doi.org/10.1136/jnnp2013-307072

2. Doherty MJ, Jayadev S, Watson NF, Konchada RS, Hallam DK 2005. Clinical implications of splenium magnetic resonance imaging signal changes. Arch Neurol 62(3): 433-437. https://doi.org/10.1001/ archneur.62.3.433
3. Conti M, Salis A, Urigo C, Canalis L, Frau S, et al. 2007. Transient focal lesion in the splenium of the corpus callosum: MR imaging with an attempt to clinical-physiopathological explanation and review of the literature. Radiol Med 112(6): 921-935. https://doi.org/10.1007/ s11547-007-0197-9

4. Puri AS, Zada G, Zarzour H, Laws E, Frerichs K. 2012. Cerebral vasospasm after transsphenoidal resection of pituitary macroadenomas: report of 3 cases and review of the literature. Neurosurgery 71(1 Suppl Operative):173-180.https://doi.org/10.1227/NEU.0b013e31824aae21

5. Kim EH, Oh MC, Kim SH. 2013. Angiographically documented cerebral vasospasm following transsphenoidal surgery for pituitary tumors. Pituitary 16(2): 260-269. https://doi.org/10.1007/s11102-0120415-7

6. Pozzati E, Frank G, Nasi MT, Giuliani G. 1987. Pituitary apoplexy, bilateral carotid vasospasm, and cerebral infarction in a 15 -year-old boy. Neurosurgery 20(1): 56-59. https://doi.org/10.1227/00006123198701000-00015

7. Popugaev KA, Savin IA, Lubnin AU, Goriachev AS, Kadashev BA, et al. 2011. Unusual cause of cerebral vasospasm after pituitary surgery. Neurol Sci 32(4): 673-680. https://doi.org/10.1007/s10072-011-0482-y

8. Nakagawa K, Serrador JM, Larose SL, Moslehi F, Lipsitz LA, et al. 2009. Autoregulation in the posterior circulation is altered by the metabolic state of the visual cortex. Stroke 40(6): 2062-2067. https:// doi.org/10.1161/STROKEAHA.108.545285

9. Strandgaard S, Paulson OB. 1984. Cerebral autoregulation. Stroke 15(3): 413-416. https://doi.org/10.1161/01.STR.15.3.413 